\title{
Comunidade de abelhas (Hymenoptera, Apoidea) e plantas em uma área do Agreste pernambucano, Brasil
}

\author{
Paulo Milet-Pinheiro ${ }^{1} \&$ Clemens Schlindwein ${ }^{2}$
}

${ }^{1}$ Programa de Pós-Graduação em Biologia Vegetal, Universidade Federal de Pernambuco. Av. Prof. Moraes Rego, s/n, 50670-901 Recife-PE. miletpinheiro@ hotmail.com

${ }^{2}$ Departamento de Botânica, Universidade Federal de Pernambuco, Av. Prof. Moraes Rego, s/n, 50670-901 Recife-PE. schlindw@ufpe.br

\begin{abstract}
Community of bees (Hymenoptera, Apoidea) and plants in an area of Agreste in Pernambuco, Brazil. The Agreste is a transition region of tropical rainforest and Caatinga in northeastern Brazil. In this region, the majority of the native Atlantic Rainforest was destroyed to give place to livestock farming. It is not known whether degraded areas maintain a diversified bee-plant community or not and which kinds of bee-plant interactions occur. The vegetation at the study site comprises pastures, ruderal vegetation and minute remanents of rainforest vegetation. Both bees and associated plants were collected monthly, from August 2001 to July 2002, sampling during two consecutive days from 5:30 am to 5:30 pm. We collected a total of 1004 bees from 79 species. Apidae was the most abundant and species-rich bee family (732 individuals, 43 species) followed by Halictidae (194 individuals and 20 spp.), Megachilidae (47 individuals and 13 spp.), Colletidae (16 individuals and 2 spp.) and Andrenidae (15 individuals and 1 sp.). Only three species of eusocial bees and five of euglossine bees were recorded, even though both groups are diversified in Neotropical rainforests. The absence of native stingless bees of the genera Plebeia, Frieseomelitta, Partamona, Scaptotrigona and Trigonisca, as well as of other euglossine bee species, is probably due to the lack of nesting sites and to the paucity of pollen and nectar resources in this disturbed area. The bees visited flowers of 87 plant species, mainly of herbs and small shrubs. Isolated rainforest trees in the pasture as well as cultivated fruit crops contributed to some extent to maintain a diversified native bee community.
\end{abstract}

KEYWORDS. Bee-plant interaction; diversity; Northeastern Brazil; species richness; pollination.

\begin{abstract}
RESUMO. Comunidade de abelhas (Hymenoptera, Apoidea) e plantas em uma área do Agreste pernambucano, Brasil. O Agreste é uma região de transição entre floresta tropical úmida e caatinga no nordeste brasileiro. Nessa região, grande parte da vegetação nativa foi desmatada para a implantação de pastagens. Não é sabido se áreas degradadas mantém uma apifauna e flora melitófila diversificada, ou quais são associações entre abelhas e plantas que ocorrem nessas áreas. A cobertura vegetal atual é composta por pastos, vegetação ruderal e restos da vegetação nativa. Abelhas e plantas por elas visitadas foram coletadas mensalmente entre agosto de 2001 e julho de 2002, durante dois dias consecutivos entre 5 h30 e 17h30. Foram coletados 1.004 indivíduos de abelhas pertencentes a 79 espécies. Apidae foi a família mais abundante e com maior riqueza de espécies (732 indivíduos e 43 espécies), seguida por Halictidae (194 indivíduos e 20 spp.), Megachilidae (47 indivíduos e 13 spp.), Colletidae (16 indivíduos e 2 spp.) e Andrenidae (15 indivíduos e 1 sp.). Foram registradas apenas três espécies de abelhas eussocais e cinco de Euglossini, dois grupos altamente diversificados nas florestas neotropicais. A ausência de abelhas sem ferrão nativas dos gêneros Plebeia, Frieseomelitta, Partamona, Scaptotrigona e Trigonisca, assim como de outras espécies de Euglossini, deve estar relacionada à falta de sítios de nidificação e à escassez de fontes de pólen e néctar nessa área degradada. Foram registradas 87 espécies de plantas melitófilas, a maioria ervas e arbustos. Árvores nativas isoladas, assim como plantas ornamentais e frutíferas cultivadas contribuem para manter parte da diversidade da comunidade de abelhas nativas.
\end{abstract}

PALAVRAS-CHAVE. Diversidade; interação abelha-planta; levantamento; Nordeste Brasileiro; polinização; riqueza de espécies.

Em 1967, Sakagami e colaboradores sugeriram uma metodologia padronizada para amostragem de comunidades de abelhas e suas plantas associadas e, a partir de então, diversos estudos seguindo essa metodologia foram realizados em diferentes regiões do Brasil. A região sul-sudeste foi, a princípio, a mais estudada com trabalhos de Sakagami \& Laroca (1971), Laroca et al. (1982), Camargo \& Mazucato (1984), Bortoli \& Laroca (1990), Wilms (1995), Schlindwein (1998), Alves-dosSantos (1999), Gonçalves \& Melo (2005) and Steiner et al. (2006). Estes levantamentos revelaram apifaunas locais com 101 até 292 espécies.

No Nordeste do Brasil, os trabalhos de Ducke $(1906,1925)$ deram início aos estudos das relações entre abelhas e plantas. No entanto, estudos da comunidade abelhas e plantas dessa região foram retomados apenas no fim do século XX, com os levantamentos padronizados de Martins (1994), Aguiar et al. (1995), Aguiar \& Martins (1997), Viana (1999), Aguiar (2003), Zanella (2003) e Aguiar \& Zanella (2005). Todos esses trabalhos salvo Martins (1994), que estudou diferentes fitofisionomias na Chapada Diamantina, foram realizados no domínio da Caatinga dos estados da Bahia, Paraíba e Rio Grande do Norte. Esses estudos, em geral, amostraram uma apifauna mais pobre do que aquela encontrada em outros ecossistemas brasileiros, mas com várias espécies endêmicas 
(Zanella 2000; Zanella \& Martins 2003). Estudos em áreas de Mata Atlântica ou de transição entre Caatinga e Mata Atlântica são escassos na região Nordeste.

No estado de Pernambuco existe apenas um levantamento de abelhas e plantas melíferas (Locatelli \& Machado 2001). Neste estudo, realizado no Parque Ecológico João Vasconcelos Sobrinho, um brejo de altitude situado no município de Caruaru, foram amostradas 101 espécies de abelhas. O objetivo do presente estudo foi conhecer a composição, diversidade e abundância de abelhas e suas relações com plantas em ChãGrande, no Agreste pernambucano. O Agreste é uma área de transição entre a Zona da Mata e o Sertão do Nordeste brasileiro, que se estende por uma vasta área dos estados da Bahia, Sergipe, Alagoas, Pernambuco, Paraíba e Rio Grande do Norte e que apresenta desde enclaves de Mata Atlântica, até formações vegetais características de Caatinga. A região encontra-se em avançado grau de degradação (estima-se que mais de $90 \%$ da área natural já foi alterada), devido principalmente a agricultura e pecuária extensiva (Velloso et al. 2002).

\section{MATERIALE MÉTODOS}

Área de estudo. $O$ estudo foi realizado no município de Chã-Grande, Agreste de Pernambuco, Brasil. Aárea de trabalho localiza-se nas redondezas da fazenda Água-Fria (S 8¹1'19.0', W 35'28' 13.6'), a cerca de $600 \mathrm{~m}$ de altitude, uma região de transição da Floresta estacional semidecídua para Mata Seca. A cobertura vegetal atual encontra-se bastante degradada, devido principalmente ao desmatamento para implantação de pastagens o que é típico em grande parte do Agreste pernambucano (Velloso et al. 2002). Na vegetação aberta, dominada por pastagens, ocorrem espécies de Caatinga, espécies ruderais de distribuição ampla, restos de vegetação nativa degradada em forma de capões, árvores isoladas e plantas frutíferas e ornamentais cultivadas. O clima da região é tropical estacional com precipitação anual entre 600 e 800 $\mathrm{mm}$. A maior concentração das chuvas ocorre entre os meses de fevereiro e junho com um longo período de seca entre os meses de julho e fevereiro (CPTEC 2007).

Amostragem de abelhas. Entre agosto de 2001 e julho de 2002 foram realizadas coletas mensais, totalizando 12 meses. Cada uma consistiu em dois dias consecutivos de coleta, totalizando 24 coletas. Nas redondezas da fazenda, foi delimitada uma área de coleta percorrida de maneira arbitrária por dois coletores das $5 \mathrm{~h} 30$ à $17 \mathrm{~h} 30$, somando 288 horas de trabalho de campo. Aárea abrangeu campo, vegetação ruderal ao longo das estradas e trilhas e restos de vegetação nativa. Abelhas foram capturadas durante a visita às flores, ou em vôo, com rede entomológica e armazenadas em câmaras mortíferas contendo acetato de etila. As plantas visitadas pelas abelhas foram coletadas e prensadas no campo.

Adicionalmente às amostras em flores, em cada mês foram coletadas abelhas presas em uma grande janela de vidro, situada no final de um terraço da casa sede da fazenda. As abelhas coletadas neste local não foram incluídas no levantamento padronizado, mas sim em uma listagem distinta que foi comparada com os resultados de coleta nas flores.

Os espécimes de abelhas foram montados em alfinetes entomológicos, secos em estufa a $40^{\circ} \mathrm{C}$ por dois dias, etiquetados, identificados, registrados no banco de dados do Grupo de "Ecologia de Abelhas e da Polinização - Plebeia" e armazenados na Coleção Entomológica da UFPE. As plantas foram depositadas no Herbário Geraldo Mariz UFP. As abelhas foram identificadas por comparação com a coleção de referência do grupo de trabalho. Grupos específicos foram identificados por Pe. J. S. Moure, D. Urban, G. A. R. Melo, A. Aguiar (todos UFPR) e F. Silveira (UFMG). O material vegetal foi identificado por M. Oliveira (UFPE), A. Laurênio (UFRPE) e R. de Cássia (IPA-PE).

Análise de dados. Para determinar a representatividade da amostragem de abelhas da comunidade foi utilizada a curva do coletor (número acumulado de espécies) (Mueller-Dumbois $\&$ Ellenberg 1974; Pielou 1975), que mostra o aparecimento de novos táxons no decorrer das coletas. O ajuste da curva lognormal à distribuição de abundância das espécies, de acordo com o modelo Preston (1948), foi realizado a partir do software Ecological Methodology (Krebs 1998). A classificação das abelhas em tribos segue Michener (2000) e em gêneros Silveira et al. (2002). Para viabilizar comparações com estudos anteriores, a família Apidae (sensu Roig-Alsina \& Michener 1993) foi dividida em Apidae corbiculados e Apidae nãocorbiculados, correspondente à família Anthophoridae de classificações anteriores.

\section{RESULTADOS}

Foram coletados ao total 1004 indivíduos, 967 em flores, pertencentes a 79 espécies, 19 tribos e 38 gêneros, representando as cinco famílias de abelhas com ocorrência no Brasil (Tabela I). Apidae foi a família com o maior número de espécies amostradas (43 spp.) devido, principalmente, ao grupo das espécies não-corbiculadas (Fig. 1a). Destaca-se ainda entre os Apidae, a pobreza de Meliponini, que teve apenas duas espécies registradas: Trigona spinipes e Melipona scutellaris. Entre os gêneros, Megachile Latreille foi o mais diversificado com 11 espécies (14\%), seguido por Centris Fabricius com oito, Augochlora Smith com sete, Xylocopa Latreille com seis, Euglossa Latreille com cinco e Dialictus Robertson e Pseudaugochlora Michener ambas com quatro espécies (Tabela I). A maioria dos outros gêneros teve representantes de apenas um ou dois táxons (73\%).

Apidae foi a família mais abundante com 732 indivíduos (446 corbiculados), seguida por Halictidae (194), Megachilidae (47), Colletidae (16) e Andrenidae (15) (Fig. 1b). Trigona spinipes, Euglossa melanotricha e Apis mellifera foram as espécies mais abundantes com 142, 122 e 115 indivíduos amostrados, respectivamente. Juntas essas três espécies corresponderam a $37,75 \%$ dos indivíduos coletados, $51,8 \%$ dos Apidae e $85 \%$ dos Apidae corbiculados (Tabela I). 

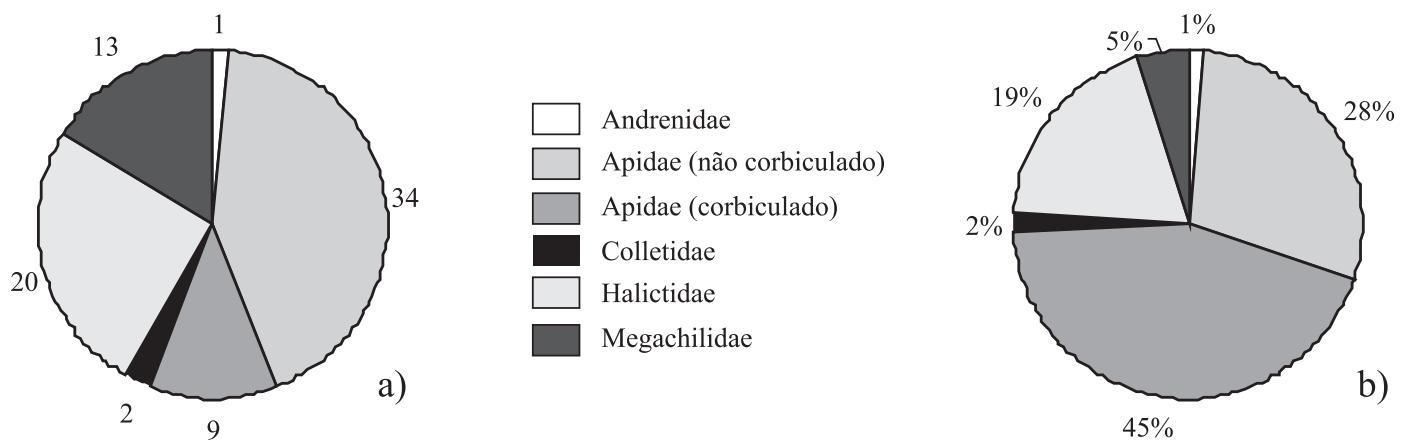

Fig. 1. Número de espécies de abelhas por família (a) e abundância relativa de indivíduos por família (b) em Chã-Grande, Pernambuco, no período de agosto de 2001 a julho de 2002.

A curva do coletor, que mede o número acumulado de espécies inéditas a cada mês, mostra uma crescente do mês de agosto de 2001 até abril de 2002, quando praticamente se estabilizou, devido ao reduzido número de novas espécies amostradas nos meses seguintes (Fig. 2).

As curvas de distribuição das espécies em classes de abundância, ajustadas à curva log-normal de Preston (1948) apresenta um corte à esquerda da moda, indicando a amostragem de poucas espécies raras (Fig. 3). A curva ajustada estimou uma riqueza de 91 espécies para a comunidade, ou seja, 11 espécies $(13,75 \%)$ a mais do que amostrado no estudo.

Na janela de vidro, foram coletadas 362 abelhas, das quais 327 indivídulos $(90,3 \%)$ eram da família Halictidae. Ao total foram registradas 29 espécies de abelhas das quais oito não foram amostradas no levantamento padronizado em flores (Tabela II).

As abelhas visitaram flores de 87 espécies vegetais, distribuídas em 38 famílias e 76 gêneros (Tabela III). As famílias com maior número de espécies visitadas por abelhas foram Asteraceae (11 espécies), Convolvulaceae (7), Fabaceae (7), Caesalpinaceae (6), Rubiaceae (5) e Verbenaceae (5). Entre as espécies vegetais cujas flores foram visitadas por abelhas, destacaram-se, Asystasia gangetica (visitada por 19 espécies), Stemodia pratensis (14 spp.), Borreria verticillata (13 spp.), Caesalpinia echinata (13 spp.), Byrsonima sericea (11 spp,),

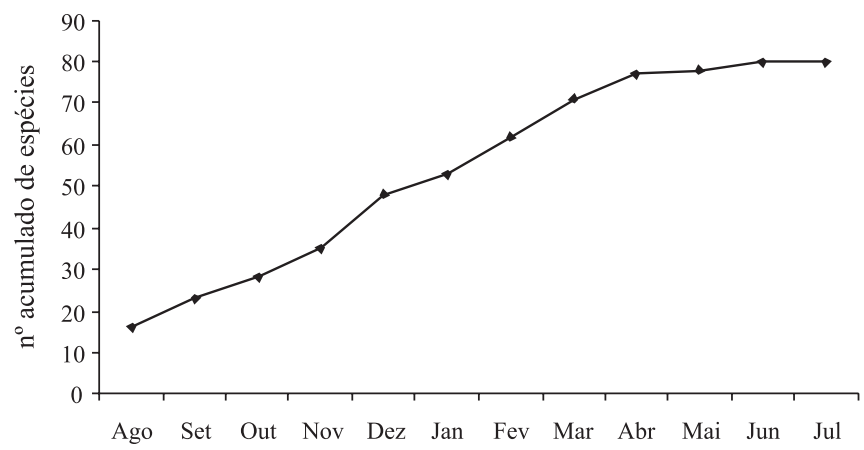

Fig. 2. Número acumulado mensal de espécies inéditas (curva do coletor) em Chã-Grande, Pernambuco, de agosto de 2001 a julho de 2002.
Ipomoea carnea (11 spp.), Tecoma stans (9 spp.), Duranta repens (8 spp.), Machaerium hirtum (8 spp.) e Vernonia scorpioides (8 spp.). As espécies de abelhas e plantas relacionadas estão listadas no Anexo I.

Arbustos e ervas foram predominantes na comunidade vegetal visitada por abelhas, sendo cada hábito representado por 29 espécies vegetais. No entanto, as flores de ervas foram visitadas por um maior número de espécies de abelhas, seguido por arbustos, árvores e lianas (Fig. 4).

\section{DISCUSSÃO}

A área de estudo apresentou uma riqueza de espécies relativamente baixa, intermediária entre regiões de Caatinga, tidas como as mais pobres entre os biomas brasileiros (Viana 1999; Zanella 2000; Zanella \& Martins 2003; Aguiar \& Zanella 2005), e outras formações como, por exemplo, Cerrado, Mata Atlântica e campos sulinos (Camargo \& Mazucato 1984; Silveira \& Campos 1995; Wilms 1995; Carvalho \& Bego 1996; Aguiar \& Martins 1997; Schlindwein 1998; Alves-dos-Santos 1999; Locatelli \& Machado 2001; Gonçalves \& Melo 2005; Steiner et al. 2006) (Fig. 5).

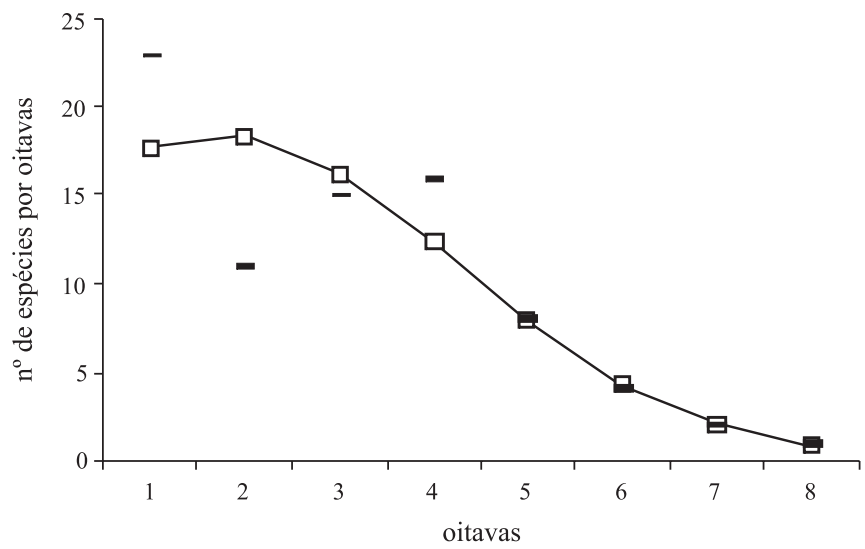

Fig. 3. Distribuição das espécies em classes de abundância (oitavas) e ajuste da curva log-normal para a comunidade de abelhas de Chã-Grande, Pernambuco. 
Tabela I. Abelhas coletadas em Chã-Grande, Pernambuco, entre agosto de 2001 e julho de 2002.

\begin{tabular}{|c|c|c|c|}
\hline \multirow[t]{2}{*}{ Espécie/família } & \multicolumn{3}{|c|}{ Número de indivíduos } \\
\hline & \multicolumn{3}{|c|}{ Machos Fêmeas Total } \\
\hline \multicolumn{4}{|l|}{ ANDRENIDAE } \\
\hline \multicolumn{4}{|l|}{ Oxainae } \\
\hline Oxaea austera Gerstaecker, 1867 & 14 & 1 & 15 \\
\hline \multicolumn{4}{|l|}{ APIDAE } \\
\hline \multicolumn{4}{|l|}{ Apinae } \\
\hline \multicolumn{4}{|l|}{ Apini ${ }^{c}$} \\
\hline Apis mellifera Linnaeus, 1758 & & 115 & 115 \\
\hline \multicolumn{4}{|l|}{ Bombini ${ }^{c}$} \\
\hline $\begin{array}{l}\text { Bombus (Fervidobombus) brevivillus Franklin, } \\
1913\end{array}$ & & 8 & 8 \\
\hline \multicolumn{4}{|l|}{ Centridini } \\
\hline Centris (Centris) aenea (Lepeletier, 1841) & 3 & 28 & 31 \\
\hline Centris (Hemisiella) tarsata Smith, 1874 & 6 & 5 & 11 \\
\hline Centris (Hemisiella) trigonoides Lepeletier, 1841 & 1 & & 1 \\
\hline Centris (Heterocentris) analis (Fabricius, 1804) & 31 & 4 & 35 \\
\hline Centris (Melacentris) sp. & 2 & & 2 \\
\hline Centris (Paracentris) hyptidis Ducke, 1908 & & 19 & 19 \\
\hline Centris (Ptilotopus) sponsa Smith, 1854 & 1 & 5 & 6 \\
\hline Centris (Trachina) fuscata Lepeletier, 1841 & 14 & 6 & 20 \\
\hline Epicharis (Epicharis) bicolor Smith, 1854 & & 1 & 1 \\
\hline Epicharis (Epicharana) flava Friese, 1900 & & 11 & 11 \\
\hline Epicharis (Hoplepicharis) sp. & & 1 & 1 \\
\hline \multicolumn{4}{|l|}{ Euglossini $^{c}$} \\
\hline Euglossa (Euglossa) cordata (Linnaeus, 1758) & 9 & 13 & 22 \\
\hline Euglossa (Euglossa) melanotricha Moure, 1967 & 72 & 50 & 122 \\
\hline Euglossa (Euglossa) securigera Dressler, 1982 & 15 & 1 & 16 \\
\hline Euglossa (Euglossa) townsendi Cockrell, 1904 & 2 & & 2 \\
\hline Eulaema (Apeulaema) nigrita Lepeletier, 1841 & 7 & 4 & 11 \\
\hline \multicolumn{4}{|l|}{ Emphorini } \\
\hline Ancyloscelis apiformis (Fabricius, 1793) & 34 & 4 & 38 \\
\hline Melitoma segmentaria (Fabricius, 1804) & 8 & 4 & 12 \\
\hline Melitoma taurea Say, 1837 & 1 & 6 & 7 \\
\hline Ptilothrix plumata Smith, 1853 & 6 & 4 & 10 \\
\hline \multicolumn{4}{|l|}{ Ericrocidini } \\
\hline Mesocheira bicolor (Fabicius, 1804) & & 2 & 2 \\
\hline Mesoplia (Mesoplia) sp.1 & 1 & & 1 \\
\hline \multicolumn{4}{|l|}{ Eucerini } \\
\hline Gaesischia sp. & 1 & & 1 \\
\hline Thygater (Thygater) analis Lepeletier, 1841 & 1 & & 1 \\
\hline \multicolumn{4}{|l|}{ Exomalopsini } \\
\hline Exomalopsis (Exomalopsis) analis Spinola, 1853 & & 7 & 7 \\
\hline $\begin{array}{l}\text { Exomalopsis (Exomalopsis) auropilosa Spinola, } \\
1853\end{array}$ & & 6 & 6 \\
\hline Exomalopsis (Exomalopsis) sp. & & 5 & 5 \\
\hline Meliponini ${ }^{c}$ & & & \\
\hline Melipona scutellaris Latreille, 1811 & & 4 & 4 \\
\hline Trigona spinipes (Fabricius, 1793) & & 142 & 142 \\
\hline Tapinotaspidini & & & \\
\hline Arhysoceble huberi (Ducke, 1908) & & 4 & 4 \\
\hline Caenonomada unicalcarata (Ducke, 1908) & & 1 & 1 \\
\hline $\begin{array}{l}\text { Paratetrapedia (Paratetrapedia) conexa (Vachal, } \\
1909 \text { ) }\end{array}$ & & 1 & 1 \\
\hline Nomadinae & & & \\
\hline Epeolini & & & \\
\hline Thalestria spinosa (Fabricius, 1804) & 1 & & 1 \\
\hline Xylocopinae & & & \\
\hline Ceratinini & & & \\
\hline Ceratina (Crewella) maculifrons Smith, 1844 & & 6 & 6 \\
\hline Ceratina (Crewella) paraguayensis Schrottky, 1907 & & 1 & 1 \\
\hline Ceratina (Ceratinula) sp. 1 & 1 & 2 & 3 \\
\hline Xylocopini & & & \\
\hline Xylocopa (Megaxylocopa) frontalis (Olivier, 1789) & & 11 & 11 \\
\hline Xylocopa (Neoxylocopa) cearensis Ducke, 1910 & & 2 & 2 \\
\hline Xylocopa (Neoxylocopa) grisescens Lepeletier, 1841 & 11 & 7 & 8 \\
\hline Xylocopa (Schonnherria) muscaria(Fabricius, 1775) & ) 2 & & 2 \\
\hline
\end{tabular}

Tabela I. Continuação.

\begin{tabular}{|c|c|c|c|}
\hline \multirow{2}{*}{ Espécie/família } & \multicolumn{3}{|c|}{ Número de indivíduos } \\
\hline & Machos & Fêmeas & Total \\
\hline Xylocopa (Neoxylocopa) ordinaria Smith, 1874 & & 16 & 16 \\
\hline Xylocopa (Neoxylocopa) suspecta Moure \& & 2 & 7 & 9 \\
\hline \multicolumn{4}{|l|}{ Camargo, 1988} \\
\hline \multicolumn{4}{|l|}{ COLLETIDAE } \\
\hline \multicolumn{4}{|l|}{ Hylaeinae } \\
\hline Hylaeus sp. 1 & & 11 & 11 \\
\hline Hylaeus sp. 2 & 5 & & 5 \\
\hline HALICTIDAE & & & \\
\hline
\end{tabular}

\section{Halictinae}

Augochlorini

Augochlora (Augochlora) esox (Vachal, 1911) $10 \quad 10$

Augochlora (Augochlora) sp. 1

Augochlora (Augochlora) sp. 2

Augochlora (Augochlora) sp. 3

Augochlora (Oxystoglossela) thalia Smith, $1879 \quad 17 \quad 17$

Augochlora (Oxystoglossela) sp. 1

Augochlora (Oxystoglossela) sp. 2

Augochlorella sp.

Augochloropsis cupreola (Cockerell, 1900)

Augochloropsis sp. 1

Augochloropsis sp. 2

Augochloropsis sp. 3

Pereirapis semiaurata (Spinola, 1851)

Pseudaugochlora graminea (Fabricius, 1804)

Pseudaugochlora pandora (Smith, 1853)

Pseudaugochlora flammula Almeida, 2008

Halictini

Dialictus (Chloralictus) opacus (Moure, 1940) $\quad 2 \quad 13 \quad 15$

Dialictus (Chloralictus) sp. 1

Dialictus (Chloralictus) sp. 2

Dialictus (Chloralictus) sp. 3

\section{MEGACHILIDAE}

\section{Anthidiini}

Dicranthidium arenarium (Ducke, 1907)

Megachilini

Coelioxys sp.

Megachile (Acentron) lentifera Vachal, 1909

Megachile (Acentron) sp. 1

Megachile (Acentron) sp. 2

Megachile (Austromegachile) susurrans Haliday, 1836

Megachile (Cressoniella) sp.

Megachile (Dactylomegachile) sp.

Megachile (Leptorachis) paulistana Schrottky, 19023

Megachile (Moureapis) apicipennis Schrottky, 19021

Megachile (Pseudocentron) sp. 1

Megachile (Pseudocentron) sp. 2

Megachile (Pseudocentron) sp. 3

\begin{tabular}{lccc}
\hline Total & 300 & 704 & 1004 \\
\hline
\end{tabular}

${ }^{c}$ Apidae corbiculados

A composição de espécies na comunidade em Chã-Grande assemelhou-se à de Cerrado onde houve, de modo geral, predominância dos Apidae não corbiculados, seguido por Halictidae, Megachilidae e Apidae corbiculados (Fig. 6). Essa predominância de Apidae não corbiculados tem sido registrada na maioria dos estudos realizados no Brasil (Fig. 6).

A representatividade relativa das diversas famílias de abelhas varia significativamente nas diferentes regiões do Brasil. A família Halictidae é mais representativa em estudos realizados no sul do Brasil (Sakagami et al. 1967; Bortoli \& Laroca 1990, 1997; Wittmann \& Hoffman 1990; Schlindwein 
$\square$ espécies de abelhas relacionadas $\square$ indivíduos relacionados $\square$ espécies vegetais

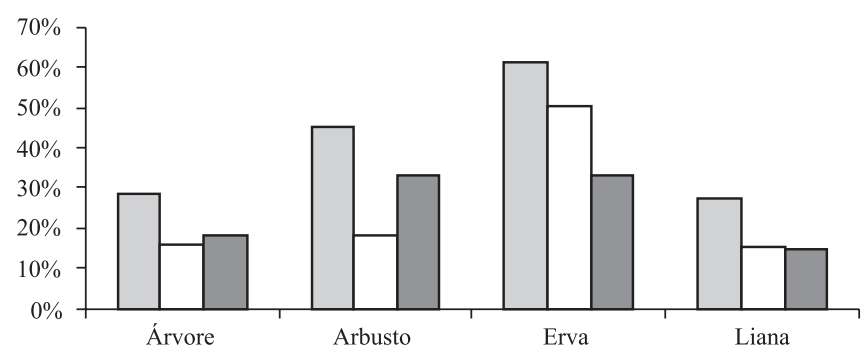

Fig 4. Percentual das espécies e indivíduos de abelhas e das espécies vegetais por hábito em Chã-Grande, Pernambuco.

1998; Alves-dos-Santos 1999), o que segue o padrão de distribuição desta família (Michener 1979; Roubik 1989). A família Megachilidae, por sua vez, não apresenta um padrão claro de diversidade nos levantamentos realizados, sendo sempre representativa nas comunidades de abelhas nas diversas regiões (Fig. 6).

As famílias Andrenidae e Colletidae são mais diversificadas nas regiões temperadas e subtropicais do sul da América do Sul (Michener 1979; Roubik 1989; Silveira et al. 2002; Schlindwein 2003) e, quando não ausentes, são pouco presentes nas comunidades estudadas na maior parte do Brasil (Fig. 6).

Os Apidae corbiculados tendem a aumentar sua diversidade em direção ao Equador, sendo mais bem representados em florestas tropicais quentes e úmidas (Michener 1979; Roubik 1989). Meliponini e Euglossini são abelhas caracteristicamente mais diversificadas em florestas úmidas (Moure 1967; Roubik 1989). A baixa riqueza de abelhas dessas duas tribos encontrada em Chã-Grande deve estar relacionada ao atual estado de degradação da vegetação que causa: 1) escassez de sítios de nidificação, uma vez que essas abelhas constroem seus ninhos em cavidades pré-existentes de troncos; 2) escassez de recursos florais, principalmente de árvores com floração em massa e 3) falta de orquídeas epífitas produtoras de perfumes florais (Roubik 1989; Ramalho 2004). Locatelli \& Machado (2001) estudando em um brejo de altitude com pouco impacto humano, a cerca de $30 \mathrm{~km}$ de Chã-Grande, encontraram cinco espécies de Meliponini e sete de Euglossini. Gonçalves e colaboradores (1996) registraram dez espécies de Meliponini e oito de Euglossini em uma área de mata secundária em Alcântara, Maranhão.

Zanella (2003) vinculou o baixo número de abelhas sem ferrão à ação humana, seja pela destruição de ninhos para obtenção de mel, seja pela eliminação ou redução da cobertura vegetal. Na Estação Ecológica do Seridó, que mesmo após vinte anos de proteção ainda continua coberta por uma Caatinga arbustiva, esse autor encontrou apenas uma espécie de Meliponini, enquanto Martins (1994), estudando em uma área mais preservada e de Caatinga arbórea amostrou 10 espécies.

Na Mata Atlântica de Pernambuco pelo menos 25 espécies de Euglossini são conhecidas e a maioria delas é dependente da floresta (Milet-Pinheiro \& Schlindwein 2005; Darrault et al. 2005). Em regiões de brejo de altitude, formações florestais úmidas, era de se esperar uma maior riqueza desse grupo, como apresentado por Locatelli \& Machado (2001). A ocorrência de apenas cinco espécies de Euglossini na área de estudo (confirmado por coletas em iscas odoríferas), no entanto, é mais similar àquela de áreas de Caatinga, onde até o momento foram registradas apenas Euglossa cordata, Eg. melanotricha e Eulaema nigrita (Zanella 2000). Eulaema nigrita e Euglossa cordata são comuns em ambientes abertos, degradados e até mesmo urbanos e não são dependentes da Mata (Silveira et al. 2002; Milet-Pinheiro \& Schlindwein 2005), enquanto

Tabela II. Abelhas coletadas na armadilha em Chã-Grande, Pernambuco, entre agosto de 2001 e julho de 2002.

\begin{tabular}{|c|c|c|c|}
\hline \multirow[t]{2}{*}{ Espécie/família } & \multicolumn{3}{|c|}{ Número de indivíduos } \\
\hline & \multicolumn{3}{|c|}{ Machos Fêmeas Total } \\
\hline \multicolumn{4}{|l|}{ ANDRENIDAE } \\
\hline \multicolumn{4}{|l|}{$\begin{array}{l}\text { Panurginae } \\
\text { Calliopsini }\end{array}$} \\
\hline Acamptopoeum prinii Holmberg, 1884* & 1 & & 1 \\
\hline \multicolumn{4}{|l|}{ APIDAE } \\
\hline \multicolumn{4}{|l|}{ Apinae } \\
\hline \multicolumn{4}{|l|}{ Apini ${ }^{c}$} \\
\hline Apis mellifera Linnaeus, 1758 & & 4 & 4 \\
\hline \multicolumn{4}{|l|}{ Euglossini ${ }^{c}$} \\
\hline Euglossa (Euglossa) cordata (Linnaeus, 1758) & 6 & & 6 \\
\hline Euglossa (Euglossa) melanotricha Moure, 1967 & 6 & & 6 \\
\hline Euglossa (Euglossa) securigera Dressler, 1982 & & 1 & 1 \\
\hline \multicolumn{4}{|l|}{ Emphorini } \\
\hline Ancyloscelis apiformis (Fabricius, 1793) & & 2 & 2 \\
\hline \multicolumn{4}{|l|}{ Meliponini ${ }^{\mathrm{c}}$} \\
\hline Trigona spinipes (Fabricius, 1793) & & 13 & 13 \\
\hline \multicolumn{4}{|l|}{ Xylocopinae } \\
\hline \multicolumn{4}{|l|}{ Ceratinini } \\
\hline Ceratina (Crewella) maculifrons Smith, 1844 & & 1 & 1 \\
\hline Ceratina (Ceratinula) sp. 1 & & 1 & 1 \\
\hline
\end{tabular}

\section{HALICTIDAE}

Halictinae

\section{Augochlorini}

Augochlora (Augochlora) esox (Vachal, 1911) $\quad 8 \quad 8$

Augochlora (Augochlora) sp. 1

Augochlora (Augochlora) sp. 4*

Augochlora (Augochlora) sp. 5*

Augochlora (Oxystoglossela) sp. 1

Augochlora (Oxystoglossela) sp. 2

Augochlora (Oxystoglossela) sp. 3*

Augochlora (Oxystoglossela) thalia Smith, 1879

Augochlorella sp. 1

Augochlorella sp. 2*

Augochloropsis sp. 1

Augochloropsis sp. 2

Augochloropsis sp. 3

Augochloropsis sp. 4*

Caenohalictus incertus (Schrottky, 1902)*

Pereirapis semiaurata (Spinola, 1851)

Pseudaugochlora graminea (Fabricius, 1804)

Pseudaugochlora pandora (Smith, 1853)

Halictini

Dialictus (Chloralictus) opacus (Moure, 1940) $19 \quad 19$

\begin{tabular}{lccc} 
Dialictus (Chloralictus) sp. 4* & 1 & 1 \\
\hline Total & 58 & 304 & 362
\end{tabular}

c Apidae corbiculados

* espécies coletadas exclusivamente na armadilha 


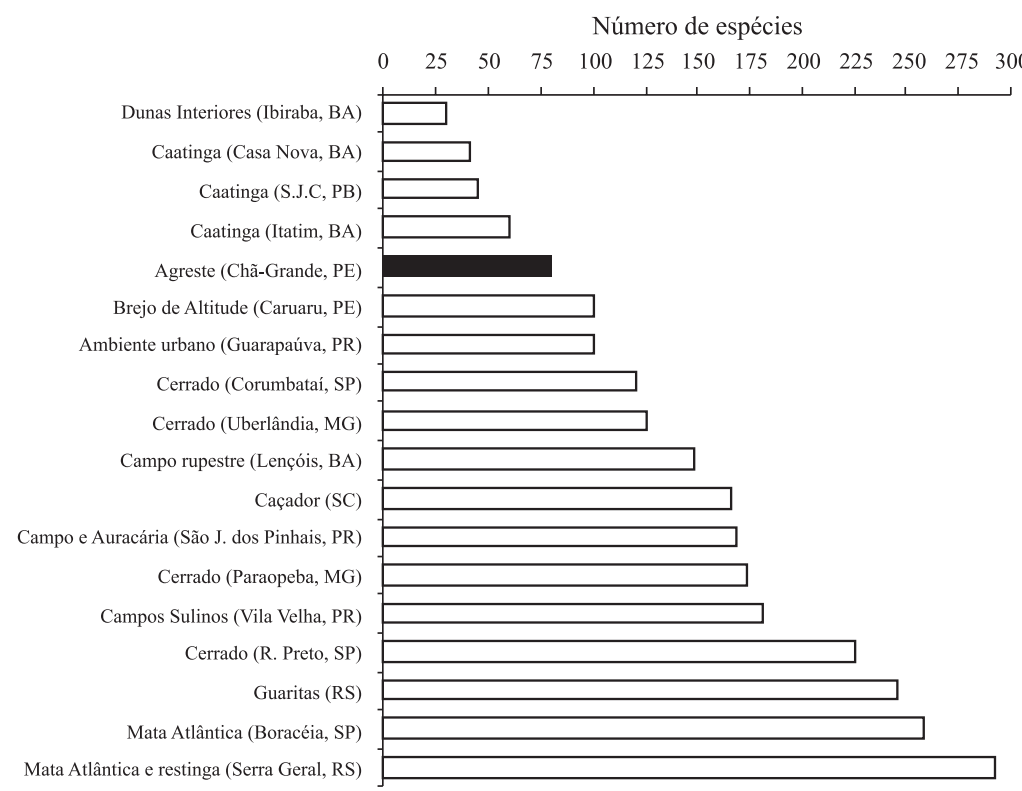

Fig 5. Riqueza de espécies de abelhas em diferentes áreas do Brasil. Fontes: São José dos Pinhais, PR (Sakagami et al. 1967); Ribeirão Preto, SP (Camargo \& Mazucato 1984); Lençóis e Casa Nova, BA (Martins 1994); Corumbataí, SP e Paraopeba, MG (Silveira \& Campos 1995); Boracéia, SP (Wilms 1995); Uberlândia, MG (Carvalho \& Bego 1996 ); São João do Cariri, PB (Aguiar \& Martins 1997); Guarapaúva, PR (Bortoli \& Laroca 1997); Guaritas, RS (Schlindwein 1998); Serra Geral, RS (Alves-dos-Santos 1999); Ibiraba, BA (Viana 1999); Caruaru, PE (Locatelli \& Machado 2001); Itatim, BA (Aguiar \& Zanella 2005), Vila Velha, PR (Gonçalves \& Melo 2005) e Caçador, SC (Steiner et al. 2006) Chã-Grande, PE (este trabalho).

Euglossa melanotricha, também comum em áreas abertas, é a espécie de Euglossini mais abundante no Cerrado (Nemésio \& Faria 2004).

A família Apidae apresentou a maior abundância relativa de indivíduos, devido, nesse caso, aos Apidae corbiculados.
As espécies Trigona spinipes, Euglossa melanotricha e Apis mellifera foram responsáveis por $37,75 \%$ dos indivíduos coletados. A maior abundância de espécies como A. mellifera e $T$. spinipes tem sido registrada em diversos outros trabalhos (Martins 1994; Carvalho \& Bego 1996; Aguiar \& Martins 1997;

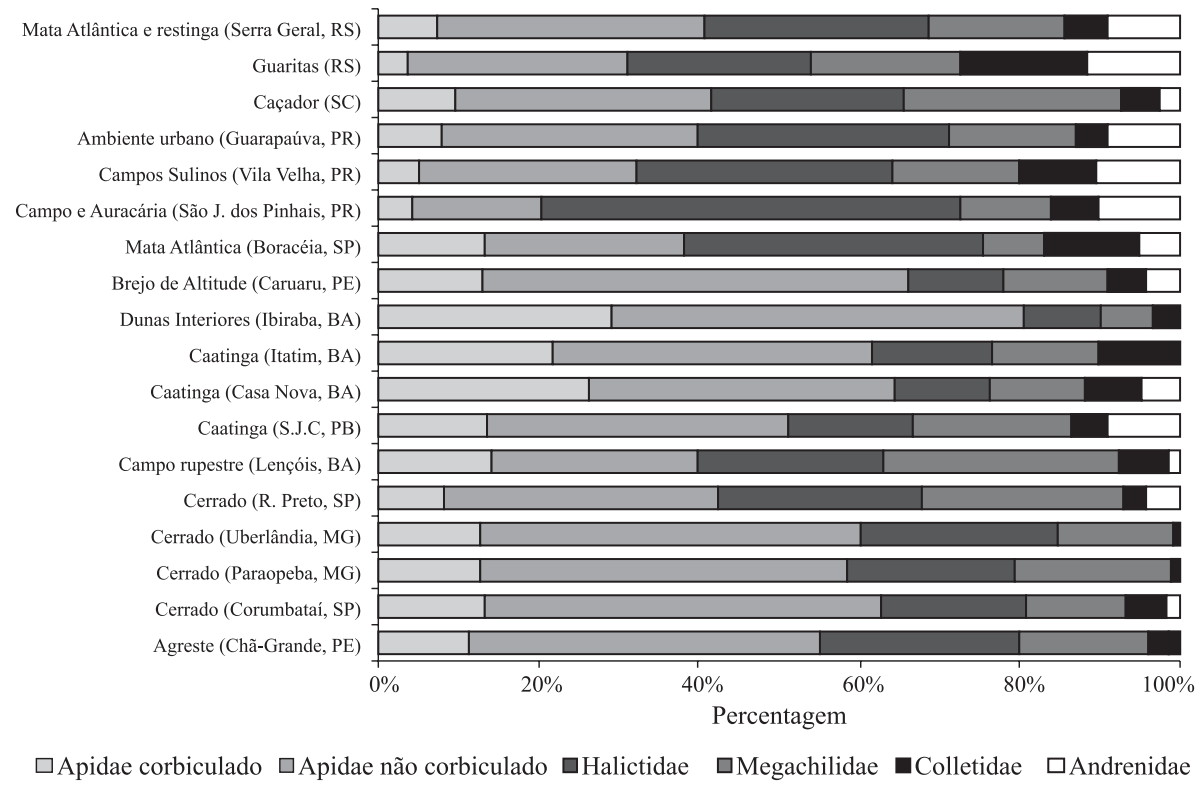

Fig. 6. Riqueza relativa de espécies em áreas com diferentes domínios vegetais do Brasil. Fontes: São José dos Pinhais, PR (Sakagami et al. 1967); Ribeirão Preto, SP (Camargo \& Mazucato 1984); Lençóis e Casa Nova, BA (Martins 1994); Corumbataí, SP e Paraopeba, MG (Silveira \& Campos 1995); Boracéia, SP (Wilms 1995); Uberlândia, MG (Carvalho \& Bego 1996 ); São João do Cariri, PB (Aguiar \& Martins 1997); Guarapaúva, PR (Bortoli \& Laroca 1997); Guaritas, RS (Schlindwein 1998); Serra Geral, RS (Alves-dos-Santos 1999); Ibiraba, BA (Viana 1999); Caruaru, PE (Locatelli \& Machado 2001); Itatim, BA (Aguiar \& Zanella 2005), Vila Velha, PR (Gonçalves \& Melo 2005) e Caçador, SC (Steiner et al. 2006) Chã-Grande, PE (este trabalho). 
Tabela III. Espécies vegetais visitadas por abelhas em Chã-Grande, Pernambuco.

\begin{tabular}{|c|c|c|c|}
\hline Família & Espécies & Hábito & Categoria \\
\hline \multirow{4}{*}{ Acanthaceae } & Asystasia gangetica G.F.W. Meyer & Erva & Ornamental \\
\hline & Hygrophila costata Nees. & Arbusto & Nativa \\
\hline & Ruellia bahiensis (Ness.) Morong. & Arbusto & Nativa \\
\hline & Thunbergia grandiflora Roxb. & Liana & Ornamental \\
\hline Amaranthaceae & Alternanthera moquinii Dusén & Arbusto & Nativa \\
\hline Amaryllidaceae & Hippeastrum psitacinum Herb. & Erva & Ornamental \\
\hline \multirow[t]{2}{*}{ Anacardiaceae } & Anacardium occidentale L. & Árvore & Cultivada \\
\hline & Spondias purpurea $\mathrm{L}$. & Árvore & Cultivada \\
\hline Annonaceae & Annona muricata $\mathrm{L}$. & Árvore & Cultivada \\
\hline Apiaceae & Coriandrum sativum $\mathrm{L}$. & Erva & Cultivada \\
\hline Apocynaceae & Allamanda blanchetii Dc. & Arbusto & Nativa \\
\hline \multirow[t]{11}{*}{ Asteraceae } & Ageratum conyzoides $\mathrm{L}$. & Erva & Nativa \\
\hline & Baccharis oxyodonta Dc. & Arbusto & Nativa \\
\hline & Baccharis trinervis (Lam.) Pers. & Arbusto & Nativa \\
\hline & Blainvillea rhomboidea Cass. & Erva & Nativa \\
\hline & Centratherum punctatum Cass. & Erva & Nativa \\
\hline & Delilea biflora (L.) Kuntz & Arbusto & Nativa \\
\hline & Sonchus oleraceus L. & Erva & Nativa \\
\hline & Tilesia baccata (L.) Pruski & Arbusto & Nativa \\
\hline & Verbesina macrophylla (Cass.) Bake & Arbusto & Nativa \\
\hline & Vernonia scorpioides (Lam.) Pers. & Erva & Nativa \\
\hline & Wedelia villosa Gardner & Erva & Nativa \\
\hline Bignoniaceae & Tecoma stans (L.) ex. Kunth. & Arbusto & Ornamental \\
\hline \multirow[t]{2}{*}{ Boraginaceae } & Cordia sellowiana Cham. & Árvore & Nativa \\
\hline & Heliotropium elongatum Murr. & Erva & Nativa \\
\hline Brassicaceae & Raphanus raphanistrum $\mathrm{L}$. & Erva & Cultivada \\
\hline \multirow[t]{6}{*}{ Caesalpiniaceae } & Caesalpinia echinata Lam. & Árvore & Nativa \\
\hline & Chamaecrista pascuorum (Benth.) Irwin \& Barneby & Erva & Nativa \\
\hline & Senna georgica Irwin \& Barneby & Arbusto & Nativa \\
\hline & Senna lechriosperma Irwin \& Barneby & Arbusto & Nativa \\
\hline & Senna siamea (Lam.) Irwin \& Barneby & Árvore & Nativa \\
\hline & Senna spectabilis Irwin \& Barneby & Arbusto & Nativa \\
\hline Combretaceae & Buchenavia sp. & Árvore & Nativa \\
\hline Commelinaceae & Commelina benghalensis $\mathrm{L}$. & Erva & Nativa \\
\hline \multirow[t]{7}{*}{ Convolvulaceae } & Ipomoea carnea Jacq. & Liana & Nativa \\
\hline & Ipomoea sp. 1 & Liana & Nativa \\
\hline & Ipomoea sp. 2 & Liana & Nativa \\
\hline & Ipomoea sp. 3 & Liana & Nativa \\
\hline & Jacquemontia densiflora (Meissn.) Hallier & Liana & Nativa \\
\hline & Jacquemontia sp. & Liana & Nativa \\
\hline & Merremia macrocalyx (Ruiz \& Pav) O'donell & Liana & Nativa \\
\hline \multirow[t]{2}{*}{ Cucurbitaceae } & Apodanthera glaziovii Cogn. & Erva & Nativa \\
\hline & Melothria fluminensis Gardner & Liana & Nativa \\
\hline Euphorbiaceae & Croton rhamnifolius H.B.K & Arbusto & Nativa \\
\hline \multirow[t]{6}{*}{ Fabaceae } & Bowdichia virgilioides H.B.K & Árvore & Nativa \\
\hline & Cajanus cajan (L.) Millesp. & Arbusto & Nativa \\
\hline & Centrosema brasilianum (L.) Benth. & Liana & Nativa \\
\hline & Crotalaria sp. & Erva & Nativa \\
\hline & Machaerium hirtum (Vell.) Stellfeld & Árvore & Nativa \\
\hline & Macroptilium latryoides (L.) Urb. & Erva & Nativa \\
\hline Fabaceae & Phaseolus vulgaris L. & Erva & Cultivada \\
\hline Liliaceae & Asparagus sp. & Erva & Ornamental \\
\hline Lythraceae & Lawsonia inermis L. & Árvore & Nativa \\
\hline \multirow[t]{3}{*}{ Malpighiaceae } & Banisteriopsis gardneriana Juss. & Arbusto & Nativa \\
\hline & Byrsonima sericea Dc. & Árvore & Nativa \\
\hline & Malpighia emarginara Sessé \& Moc. Ex. Dc. & Arbusto & Cultivada \\
\hline \multirow{3}{*}{ Malvaceae } & Malvaviscus arboreus Cav. & Arbusto & Ornamental \\
\hline & Pavonia cancelata Cav. & Erva & Nativa \\
\hline & Sida glomerata Cav. & Arbusto & Nativa \\
\hline
\end{tabular}


Tabela III. Continuação.

\begin{tabular}{|c|c|c|c|}
\hline Família & Espécies & Hábito & Categoria \\
\hline Melastomataceae & Microlicia sp. & Erva & Nativa \\
\hline Mimosaceae & Calliandra surinamensis Benth. & Arbusto & Ornamental \\
\hline \multirow[t]{4}{*}{ Myrtaceae } & Eugenia uniflora $\mathrm{L}$. & Arbusto & Cultivada \\
\hline & Jambosa aquae Roxb. & Árvore & Cultivada \\
\hline & Jambosa malaccensis Dc. & Árvore & Cultivada \\
\hline & Psidium guayava $\mathrm{L}$. & Árvore & Cultivada \\
\hline Nyctaginaceae & Bougainvillea spectabilis Willd. & Liana & Ornamental \\
\hline Oxalidaceae & Averrhoa carambola $\mathrm{L}$. & Árvore & Cultivada \\
\hline Passifloraceae & Passiflora cincinnata Mast. & Liana & Nativa \\
\hline Polygonaceae & Antigonon leptopus Hook. \& Arn. & Liana & Ornamental \\
\hline Pontederiaceae & Eichornia crassipes (Mart.) Solms-Laubach & Erva & Nativa \\
\hline \multirow[t]{5}{*}{ Rubiaceae } & Borreria allata Dc. & Erva & Nativa \\
\hline & Borreria verticillata $\mathrm{L}$. & Erva & Nativa \\
\hline & Diodia radula (R et. S.) Cham. et Schlecht & Erva & Nativa \\
\hline & Diodia sp. & Erva & Nativa \\
\hline & Ixora coccinea $\mathrm{L}$. & Arbusto & Ornamental \\
\hline Rutaceae & Citrus sinensis (L.) Osbeck & Arbusto & Cultivada \\
\hline Sapindaceae & Cupania revoluta Radlk. & Árvore & Nativa \\
\hline \multirow[t]{2}{*}{ Scrophulariaceae } & Angelonia cornigera Hook. & Erva & Nativa \\
\hline & Stemodia pratensis (Aubl.) C. P. Cowar & Erva & Nativa \\
\hline Solanaceae & Solanum paniculatum $\mathrm{L}$. & Arbusto & Nativa \\
\hline Sterculiaceae & Waltheria indica $\mathrm{L}$. & Arbusto & Nativa \\
\hline Turneraceae & Turnera subulata Smith & Arbusto & Nativa \\
\hline \multirow{5}{*}{ Verbenaceae } & Duranta repens L. & Arbusto & Ornamental \\
\hline & Ghinia sp. & Erva & Nativa \\
\hline & Lantana caatingensis Mold. & Arbusto & Nativa \\
\hline & Lippia alba (Mill.) N. E. Br. Ex Britton \& Wilson & Arbusto & Cultivada \\
\hline & Priva bahiensis Dc. & Erva & Nativa \\
\hline
\end{tabular}

Viana 1999; Zanella 2003; Aguiar \& Zanella 2005) e deve estar associada ao fato de Trigona spinipes construir ninhos expostos, independente de cavidades pré-existentes, e Apis mellifera ser uma abelha domesticada. Infelizmente, alguns autores excluíram $A$. mellifera de suas amostras por consideralá uma espécie introduzida (Camargo \& Mazucato 1984; Bortoli \& Laroca 1990, 1997; Silveira \& Campos 1995, entre outros), o que dificulta a avaliação da apifauna local e comparação com resultados de outros estudos. Euglossa melanotricha, por sua vez, é uma espécie comum do Cerrado e áreas abertas que nidifica no solo (Augusto \& Garófalo 2007).

A estabilidade atingida pela curva do coletor (Fig. 2) indica a representatividade da amostra da comunidade, no entanto, tanto a curva ajustada log-normal, quanto o corte à esquerda da moda (Fig. 3) mostra que somente poucas espécies raras foram amostradas. As oito espécies adicionais coletadas na janela de vidro (Tabela II) indicam uma apifauna local mais rica. Locatelli et al. (2004) verificaram que após a curva atingir uma suposta estabilidade (no primeiro ano de campo), coletas subseqüentes (segundo ano) acresceram a amostragem em cerca de 20 espécies. Um aumento do esforço de coleta, portanto, poderia incrementar a riqueza de espécies da comunidade de abelhas em Chã-Grande.

A flora apícola da área de estudo caracterizou-se pela riqueza de espécies de Asteraceae, comuns em áreas abertas com vegetação herbácea. Vários levantamentos apifaunísticos em diferentes locais do Brasil mostraram a importância de Asteraceae como fornecedoras de recursos florais (Camargo \& Mazucato 1984; Cure et al. 1992; Martins 1994; Carvalho \& Bego 1997; Schlindwein 1998; Alves-dos-Santos 1999; Locatelli \& Machado 2001). Na Caatinga, contudo, as compostas não estão entre as principais fornecedoras de recursos florais para abelhas (Aguiar et al. 1995; Aguiar 2003).

Nosso estudo demonstra que indivíduos isolados de árvores nativas apresentaram um papel fundamental para a manutenção da apifauna local. Byrsonima sericea (Muricí), por exemplo, garante recursos indispensáveis para pelo menos 11 espécies de abelhas. A importância da $B$. sericea se torna ainda mais evidente porque Paratetrapedia conexa e três espécies do gênero Epicharis foram encontradas visitando unicamente flores dessa árvore, à procura de óleos florais. Árvores isoladas de Machaerium hirtum, Bowdichia virgilioides (Fabaceae) e Caesalpinia echinata (Pau-Brasil), árvores com floração do tipo big bang, oferecem néctar e pólen para pelo menos 19 espécies de abelhas nativas.

Além de utilizarem plantas nativas, muitas abelhas também visitaram flores de plantas frutíferas cultivadas, como as da acerola (Malpighia emarginata) e de maracujá-amarelo (Passiflora edulis), e flores de plantas ornamentais, como Thunbergia grandiflora, Asystasia gangetica (Acanthaceae) 
e Tecoma stans (Bignoniaceae). Asystasia gangetica, apesar de ser uma planta da Ásia tropical, foi a mais procurada por abelhas, um total de 19 espécies, sendo Euglossa townsendi e Pseudaugochlora flammula sp. registradas apenas nas flores dessa planta.

Diversas espécies de abelhas nativas são polinizadores efetivos das plantas frutíferas como, por exemplo, Centris spp. de acerola e Xylocopa spp. de maracujá. A presença dessas abelhas é de fundamental importância para garantir uma boa produtividade das frutíferas. Nesse caso, a ausência de recursos florais nativos pode, pelo menos em parte, ser compensada por espécies cultivadas selecionadas. O manejo adequado e ecologicamente sustentável de cultivares, plantas ornamentais e da vegetação do entorno é, nesse contexto, uma alternativa para garantir alta produtividade dos cultivares e ao mesmo tempo a manutenção da apifauna nativa.

Agradecimentos. Agradecemos a Renildo Milet pela estrutura física e logística na Fazenda Água-Fria, Carlos Eduardo Pinto, Ana Gabriela D. Bieber e David Morais pela ajuda no trabalho de campo, André Laurênio, Marcondes Oliveira e Rita de Cássia pela colaboração na identificação do material botânico e a Jesus Santiago Moure, Danúncia Urban, Gabriel Melo, Antonio Aguiar e Fernando Silveira pela ajuda na identificação das abelhas. Ao CNPq pelo fornecimento das bolsas.

\section{REFERÊNCIAS}

Aguiar, C. M. 2003. Utilização de recursos florais por abelhas (Hymenoptera, Apoidea) em uma área de Caatinga (Itatim, Bahia, Brasil). Revista Brasileira de Zoologia 20: 457-467.

Aguiar, C. M. L.; C. F. Martins \& A. Moura. 1995. Recursos florais utilizados por abelhas (Hymenoptera, Apoidea) em área de Caatinga (São João do Cariri, Paraíba). Revista Nordestina de Biologia 10: $101-117$.

Aguiar, C. M. L. \& C. F. Martins. 1997. Abundância relativa, diversidade e fenologia de abelhas (Hymenoptera, Apoidea) na caatinga, São João do Cariri, Paraíba, Brasil. Iheringia, Série Zoologia 83: $151-163$.

Aguiar, C. M. L. \& F. C. V. Zanella. 2005. Estrutura da comunidade de abelhas (Hymenoptera: Apoidea: Apiformes) de uma área na margem do domínio caatinga (Itatim, BA). Neotropical Entomology 34: 15-24.

Alves-dos-Santos, I. 1999. Abelhas e plantas melíferas da mata atlântica, restinga e dunas do litoral norte do estado do Rio Grande do Sul, Brasil. Revista Brasileira de Entomologia 43: 191-223.

Augusto, S. C. \& C. A. Garófalo. 2007. Nidificação de Euglossa (Euglossa) melanotricha (Hymenoptera: Apidae) no solo do Cerrado. Neotropical Entomology 36: 153-156.

Bortoli, C. \& S. Laroca. 1990. Estudo biocenótico em Apoidea (Hymenoptera) de uma área restrita em São José dos Pinhais (PR, Sul do Brasil), com notas comparativas. Dusenia 15: 1-112.

Bortoli, C. \& S. Laroca 1997. Melissocenologia no terceiro planalto Paranaense. I: Abundância relativa das abelhas silvestres (Apoidea) de um biótopo urbano de Guarapaúva (PR, Brasil). Acta Biológica Paranaense 26: 51-86.

Camargo, J. M. F. \& M. Mazucato. 1984. Inventário da apifauna e flora apícola de Ribeirão Preto, SP, Brasil. Dusenia 14: 55-87.

Carvalho, A. M. C. \& L. R. Bego. 1996. Studies on Apoidea fauna of cerrado vegetation at the Panga Ecological Reserve, Uberlândia, MG, Brazil. Revista Brasileira de Entomologia 40: 147-156.

Carvalho, A. M. C. \& L. R. Bego. 1997. Exploitation of avaible resources by bee fauna (Apoidea-Hymenoptera) in the Reserva Ecológica do Panga, Uberlândia, state of Minas Gerais, Brazil. Revista Brasileira de Entomologia 41: 101-107.
CPTEC, 2007. Centro de previsão de tempos e estudos climáticos. Disponível on-line em http://www.cptec.inpe.br. Acesso em: 20/ $10 / 2007$.

Cure, J. R.; M. Thiengo; F. A. Silveira \& L. B. Rocha. 1992. Levantamento da fauna de abelhas silvestres na "Zona da Mata" de Minas Gerais. III. Mata secundária na região de Viçosa (Hymenoptera, Apoidea). Revista Brasileira de Zoologia 9: 223-239.

Darrault, R. O.; P. Medeiros; E. Locatelli; A. V. Lopes \& C. Schlindwein. 2005. Abelhas Euglossini (Hymenoptera Apidae) no centro de endemismo Pernambuco, v. 1, p. 239-256. In: K. Pôrto, J. AlmeidaCortez \& M. Tabarelli (Orgs.). Diversidade Biológica e Conservação da Floresta Atlântica ao Norte do Rio São Francisco. $1^{a}$ ed. Brasília: Ministério do Meio Ambiente, 363 p.

Ducke, A. 1906. Neue Beobachtungen über die bienen der Amazonslander. Allgemeine Zeitschrift für Entomologie 2: 51-60.

Ducke, A. 1925. Die stachellosen Biene Brasiliens. Zoologisches Jahrbuch, Abteilung für Systematiks 49: 335-448.

Gonçalves, S. J. M.; M. Rêgo \& A. Araújo. 1996. Abelhas sociais (Hymenoptera:Apidae) e seus recursos florais em uma região de mata secundária, Alcântara, MA, Brasil. Acta Amazônica 26: 5568.

Gonçalves, R. B. \& G. A. R. Melo. 2005. A comunidade de abelhas (Hymenoptera, Apidae s. 1) em uma área restrita de campo natural no Parque Estadual de Vila Velha, Paraná: diversidade, fenologia e fontes florais de alimento. Revista Brasileira de Entomologia 49: $557-571$.

Krebs, C. J. 1998. Programs for Ecological Methodology. Second edition. Versão 5.1. University of British Columbia, Vancouver.

Laroca, S. J. R. Cure \& C. Bortoli. 1982. A associação das abelhas silvestres (Hymenoptera, Apoidea) de uma área restrita no interior da cidade de Curitiba (Brasil): uma abordagem biocenótica. Dusenia 13: $93-117$.

Locatelli, E. \& I. C. S. Machado. 2001. Bee diversity and their floral resources in a fragment of a tropical altitudinal wet Forest ("Brejos de Altitude") in Northeastern Brazil. Acta Horticulturae 561: $317-325$.

Locateli, E.; I. C. S. Machado \& P. Medeiros. 2004. Riqueza de abelhas e a flora apícola em um fragmento da mata serrana (Brejo de Altitude) em Pernambuco, Nordeste do Brasil, p. 153-177. In: K. C. Pôrto, J. J. P. Cabral \& M. Tabarelli (Orgs.). Brejos de altitude em Pernambuco e Paraíba - História Natural, Ecologia e Conservação. Brasília/DF: Ministério do Meio Ambiente, 324 p.

Martins, C. F. 1994. Comunidade de abelhas (Hymenoptera, Apoidea) da caatinga e do cerrado com elementos de campo rupestre do estado da Bahia, Brasil. Revista Nordestina de Biologia 9: 225257.

Michener, C. D. 1979. Biogeography of the bees. Annals of the Missouri Botanical Gardens 66: 277-347.

Michener, C. D. 2000. The bees of the world. Baltimore, The Johns Hopkins University Press, xiv +913 p.

Milet-Pinheiro, P. \& C. Schlindwein. 2005. Do euglossine males (Apidae, Euglossini) leave tropical rainforest to collect fragrances in sugarcane monocultures? Revista Brasileira de Zoologia 22: $853-858$.

Moure, J. S. 1967. A check-list of the known euglossine bees (Hymenoptera, Apidae). Atas do Simpósio sobre a Biota Amazônica 5: 395-415.

Mueller-Dumbois, D. \& H. Ellenberg. 1974. Aims and methods of vegetation ecology. John Wiley and Sons, New York, 547 p.

Nemésio, A. \& L. R. R. Faria. 2004. First assessment of orchid bee fauna (Hymenoptera: Apidae: Apini: Euglossina) of Parque Estadual do Rio Preto, a cerrado area in southeastern Brazil. Lundiana 5: 113-117.

Pielou, E. C. 1975. Ecological diversity. John Wiley and sons, New York, viii $+165 \mathrm{p}$.

Preston, F. W. 1948. The commonness and rarity of species. Ecology 29: $254-283$.

Ramalho, M. 2004. Stingless bees and mass flowering trees in the canopy of Atlantic Forest: a tight relationship. Acta Botânica 
Brasílica 18: 37-47.

Roig-Alsina, A. \& C. D. Michener. 1993. Studies of the phylogeny and classification of long-tongued bees (Hymenoptera: Apoidea). University of Kansas Bulletin 55: 124-162.

Roubik, D. W. 1989. Ecology and natural history of tropical bees. Cambridge University Press, New York, X +514 p.

Sakagami, S. F.; S. Laroca \& J. S. Moure. 1967. Wild bee biocenotics in São José dos Pinhais (PR), South Brazil - Preliminary report. Journal of the Faculty of Hokkaido University, Series VI, Zoology 16: 252-291.

Sakagami, S. F. \& S. Laroca. 1971. Relative abundance, phenology and flower visits of Apidae bees in eastern Paraná, southern Brazil (hymenoptera, Apidae). Kontyu 39: 217-230.

Schlindwein, C. 1998. Frequent oligolecty characterizing a diverse beeplant community in a xerophytic bushland of subtropical Brazil. Studies on Neotropical Fauna and Environmental 33: 4659.

Schlindwein, C. 2003. Panurginae (Hymenoptera, Andrenidae) in Northeastern Brazil, p. 217-222. In: G. A. R. Melo \& I. Alves-dosSantos (Eds.). Apoidea Neotropica: Homenagem aos 90 Anos de Jesus Santiago Moure. Criciúma: Editora UNESC, xvi + 320 p.

Silveira, F. A. \& M. J. O. Campos. 1995. A melissofauna de Corumbataí (SP) e Paraopeba (MG) e uma análise da biogeografia das abelhas do cerrado brasileiro (Hymenoptera, Apoidea). Revista Brasileira de Entomologia 39: 371-401.

Silveira, F. A.; G. A. R. Melo \& E. A. B. Almeida. 2002. Abelhas brasileiras - sistemática e indentificação. Editora Eletrônica Composição e Arte, Belo Horizonte. 253 p.

Steiner, J.; B. Harter-Marques; A. Zillikens \& E. P. Feja. 2006. Bees of
Santa Catarina Island, Brazil - a first survey and checklist (Insecta: Apoidea). Zootaxa 1220: 1-18.

Velloso, A. L.; E. V. S. Sampaio \& F. G. L. Pareyn. 2002. Ecorregiões propostas para o Bioma Caatinga. Associação Plantas do Nordeste, Instituto de Conservação Ambiental e The Nature Conservancy do Brasil, Recife. 76 p.

Viana, B. F. 1999. A comunidade de abelhas (Hymenoptera: Apoidea) das dunas interiores do Rio São Francisco, Bahia, Brasil. Anais da Sociedade Entomológica do Brasil 28: 635-645.

Zanella, F. C. V. 2000. The bees of the caatinga (Hymenoptera, Apoidea, Apiformes): a species list and comparative notes regarding their distribution. Apidologie 31: 579-592.

Zanella, F. C. V. 2003. Abelhas da Estação Ecológica do Seridó (Serra Negra do Norte, RN): aportes ao conhecimento da diversidade, abundância e distribuição espacial das espécies da caatinga, p. 231240. In: G. A. R. Melo \& I. Alves-dos-Santos (Eds.). Apoidea Neotropica: Homenagem aos 90 anos de Jesus Santiago Moure. Criciúma: Editora UNESC, xvi +320 p.

Zanella, F. C. V. \& C. F. Martins. 2003. Abelhas da caatinga: biogeografia, ecologia e conservação, 75-134. In: I. R. Leal, M. Tabarelli \& J. M. C. Silva, (Eds.). Ecologia e conservação da caatinga. Editora Universitária, UFPE, Recife, xvi + 804 p.

Wilms, W. 1995. Die Bienenfauna im Küstenregenwald Brasiliens und ihre Beziehungen zu Blütenpflanzen: Fallstudie Boracéia, São Paulo. Tese de doutorado, Universidade de Tübingen, Alemanha, 219 p.

Wittmann, D. \& Hoffman, M. 1990. Bees of Rio Grande do Sul, Southern Brazil (Insecta, Hymenoptera, Apoidea). Iheringia Série Zoologia 70: $17-43$.

Anexo I. Relação das plantas procuradas por cada espécie de abelha amostrada em Chã-Grande, Pernambuco.

\section{ESPÉCIE/FAMÍLIA}

\section{ANDRENIDAE}

Oxaea austera - Acanthaceae: Asystasia gangetica; Caesalpinaceae: Caesalpinia echinata; Senna georgica; Fabaceae: Centrosema brasilianum.

\section{APIDAE}

Ancyloscelis apiformis - Acanthaceae: Ruellia bahiensis; Bignoniaceae: Tecoma stans; Convolvulaceae: Ipomoea carnea, Ipomoea sp.2, Jacquemontia densiflora, Merremia macrocalyx; Verbenaceae: Duranta repens.

Apis mellifera - Acanthaceae: Asystasia gangetica, Hygrophila costata, Thunbergia grandiflora; Anacardiaceae: Anacardium occidentale, Spondias purpurea; Asteraceae: Ageratum conyzoides, Baccharis oxyodonta, Centratherum punctatum, Sonchus oleraceus, Tilesia baccata, Verbesina macrophyla, Vernonia scorpioides; Boraginaceae: Cordia sellowiana; Caesalpinaceae: Caesalpinia echinata; Commelinaceae: Commelina benghalensis; Convolvulaceae: Ipomoea sp.3, Jacquemontia sp.; Euphorbiaceae: Croton rhamnifolius; Fabaceae: Bowdichia virgilioides, Machaerium hirtum, Phaseolus vulgaris; Mimosaceae: Calliandra surinamensis; Myrtaceae: Eugenia uniflora, Jambosa malaccensis, Psidium guayava; Oxalidaceae: Averrhoa carambola; Polygonaceae: Antigonon leptopus; Rubiaceae: Borreria alata, Diodia sp., Borreria verticilata; Sapindaceae: Cupania revoluta; Schrophulariaceae: Stemodia pratensis; Turneraceae: Turnera subulata; Verbenaceae: Lippia alba.

Arhysoceble huberi - Asteraceae: Centratherum punctatum; Turneraceae: Turnera subulata.

Bombus (Fervidobombus) brevivillus - Caesalpinaceae: Caesalpinia echinata; Fabaceae: Machaerium hirtum; Solanaceae: Solanum paniculatum; Verbenaceae: Lantana caatingensis.

Caenonomoda unicalcarata - Apiaceae: Coriandrum sativum.

Centris (Centris) aenea - Caesalpinaceae: Caesalpinia echinata; Fabaceae: Bowdichia virgilioides; Malpighiaceae: Byrsonima sericea, Malpighia emarginata; Verbenaceae: Duranta repens.

Centris (Heterocentris) analis - Bignoniaceae: Tecoma stans; Caesalpinaceae: Caesalpinia echinata; Convolvulaceae: Ipomoea carnea; Fabaceae: Cajanus cajan, Machaerium hirtum; Malpighiaceae: Byrsonima sericea; Malpighia emarginata; Verbenaceae: Duranta repens.

Centris (Trachina) fuscata - Caesalpinaceae: Caesalpinia echinata; Fabaceae: Machaerium hirtum; Macroptilium latrynoides; Malpighiaceae: Byrsonima sericea, Malpighia emarginata; Schrophulariaceae: Angelonia cornigera.

Centris (Paracentris) hyptidis - Fabaceae: Centrosema brasilianum.

Centris (Melacentris) sp. - Scrophulariaceae: Stemodia pratensis.

Centris (Ptilotopus) sponsa - Caesalpinaceae: Caesalpinia echinata, Senna spectabilis; Malpighiaceae: Byrsonima sericea.

Centris (Hemisiella) tarsata - Bignoniaceae: Tecoma stans; Caesalpinaceae: Caesalpinia echinata; Malpighiaceae: Byrsonima sericea, Malpighia emarginata; Verbenaceae: Duranta repens.

Centris (Hemisiella) trigonoides - Verbenaceae: Duranta repens.

Ceratina (Crewella) maculifrons - Bignoniaceae: Tecoma stans; Convolvulaceae: Ipomoea carnea; Turneraceae: Turnera subulata Ceratina (Crewella) paraguayensis - Verbenaceae: Stemodia pratensis. 
Anexo I. Continuação.

ESPÉCIE/FAMÍLIA

Ceratina (Ceratinula) sp. - Rubiaceae: Borreria verticilata.

Epicharis (Epicharis) bicolor - Malpighiaceae: Byrsonima sericea.

Epicharis (Epicharana) flava - Malpighiaceae: Byrsonima sericea.

Epicharis (Hoplepicharis) sp. - Malpighiaceae: Byrsonima sericea.

Euglossa (Euglossa) cordata - Acanthaceae: Asystasia gangetica; Bignoniaceae: Tecoma stans; Lythraceae: Lawsonia inermis; Verbenaceae: Duranta repens.

Euglossa (Euglossa) melanothrica - Acanthaceae: Asystasia gangetica; Annonaceae: Annona muricata; Asteraceae: Baccharis trinervis, Centratherum punctatum, Vernonia scorpioides; Bignoniaceae: Tecoma stans; Convolvulaceae: Ipomoea carnea; Ipomoea sp.3; Fabaceae: Centrosema brasilianum, Macroptilium latrynoides; Solanaceae: Solanum paniculatum; Verbenaceae: Duranta repens, Ghinia sp.

Euglossa (Euglossa) securigera - Acanthaceae: Asystasia gangetica, Thunbergia grandiflora; Fabaceae: Macroptilium latrynoides.

Euglossa (Euglossa) townsendi - Acanthaceae: Asystasia gangetica.

Eulaema (Apeulaema) nigrita - Acanthaceae: Asystasia gangetica; Apocynaceae: Allamanda blanchetti; Asteraceae: Tilesia baccata; Fabaceae: Macroptilium latrynoides; Mimosaceae: Caliandra surinamensis.

Exomalopsis (Exomalopsis) analis - Asteraceae: Vernonia scorpioides; Scrophulariaceae: Stemodia pratensis; Solanaceae: Solanum paniculatum.

Exomalopsis (Exomalopsis) auropilosa - Anacardiaceae: Anacardium occidentale; Fabaceae: Machaerium hirtum; Scrophulariaceae: Stemodia pratensis.

Exomalopsis (Exomalopsis) sp. - Acanthaceae: Asystasia gangetica; Asteraceae: Sonchus oleraceus; Tilesia baccata; Scrophulariaceae: Stemodia pratensis; Solanaceae: Solanum paniculatum;

Gaesischia sp. - Asteraceae: Vernonia scorpioides.

Melipona scutellaris - Caesalpinaceae: Caesalpinia echinata.

Melitoma segmentaria - Convolvulaceae: Ipomoea carnea, Ipomoea sp.2, Ipomoea sp.3.

Melitoma taurea - Convolvulaceae: Ipomoea carnea, Ipomoea sp. 3

Mesocheria bicolor - Bignoniaceae: Tecoma stans.

Mesoplia (Mesoplia) sp.1 - Verbenaceae: duranta repens.

Paratetrapedia conexa - Malpighiaceae: Byrsonima sericea.

Ptilothrix plumata - Asteraceae: Wedelia villosa; Convolvulaceae: Ipomoea sp.2; Malvaceae: Pavonia cancellata.

Thalestria spinosa - Asteraceae: Tilesia baccata.

Thygater (Thygater) analis - Convolvulaceae: Merremia macrocalyx.

Trigona spinipes - Acanthaceae: Asystasia gangetica, Thunbergia grandiflora; Anacardiaceae: Spondias purpurea, Apiaceae: Coriandrum sativum; Asteraceae: Sonchus oleraceus, Vernonia scorpioides, Wedelia villosa; Bignoniaceae: Tecoma stans; Caesalpinaceae: Caesalpinia echinata, Senna spectabilis; Combretaceae: Buchenavia sp.; Commelinaceae: Commelina benghalensis; Convolvulaceae: Ipomoea carnea, Ipomoae sp. 2, Merremia macrocalyx; Euphorbiaceae: Croton rhamnifolius; Fabaceae: Bowdichia virgilioides, Cajanus cajan, Crotalaria sp., Machaerium hirtum, Phaseoulus vulgaris; Liliaceae: Asparagus sp.; Malpighiaceae: Banisteropsis gardneriana, Byrsonima sericea, Malpighia emarginata; Mimosaceae: Calliandra surinamensis; Myrtaceae: Eugenia uniflora, Jambosa malaccensis, Psidium guayava; Nyctaginaceae: Bougainvillea spectabilis; Oxalidaceae: Averrhoa carambola; Polygonaceae: Antigonon leptopus; Pontederiaceae: Eichornia crassipes; Rubiaceae: Borreria alata, Borreria verticilata, Ixora coccinea; Rutaceae: Citrus sinensis; Solanaceae: Solanum paniculatum; Turneraceae: Turnera subulata.

Xylocopa (Neoxylocopa) cearensis - Fabaceae: Machaerium hirtum, Macroptilium latrynoides.

Xylocopa (Megaxylopa) frontalis - Acanthaceae: Thunbergia grandiflora; Caesalpinaceae: Senna spectabilis; Fabaceae: Bowdichia virgilioides, Phaseoulus vulgaris.

Xylocopa (Neoxylocopa) grisescens - Acanthaceae: Thunbergia grandiflora; Fabaceae: Phaseoulus vulgaris.

Xylocopa (Neoxylocopa) ordinaria - Acanthaceae: Asystasia gangetica; Amaryllidaceae: Hippeastrum psittacinum; Fabaceae: Centrosema brasilianum, Macroptilium latrynoides, Phaseoulus vulgaris; Malvaceae: Malvaviscus arboreus; Verbenaceae: Lantana caatingensis.

Xylocopa (Neoxylocopa) suspecta - Acanthaceae: Asystasia gangetica; Bignoniaceae: Tecoma stans; Caesalpinaceae: Senna spectabilis; Fabaceae: Bowdichia virgilioides.

\section{COLLETIDAE}

Hylaeus sp.1 - Rubiaceae: Borreria alata, Borreria verticilata.

Hylaeus sp.2 - Acanthaceae: Asystasia gangetica; Rubiaceae: Borreria verticilata.

\section{HALICTIDAE}

Augochlora (Augochlora) sp. 1 - Convolvulaceae: Ipomoea carnea; Rubiaceae: Borreria verticilata.

Augochlora (Augochlora) sp. 2 - Acanthaceae: Asystasia gangetica.

Augochlora (Augochlora) sp. 3 - Convolvulaceae: Ipomoea carnea.

Augochlora (Oxystoglossela) esox - Acanthaceae: Thunbergia grandiflora; Caesalpinaceae: Caesalpinia echinata; Convolvulaceae: Ipomoea carnea; Turneraceae: Turnera subulata.

Augochlora (Oxystoglossela) sp. 1 - Acanthaceae: Thunbergia grandiflora.

Augochlora (Oxystoglossela) sp. 2 - Rubiaceae: Borreria verticilata.

Augochlora (Oxystoglossela) thalia - Asteraceae: Baccharis oxyodonta, Blainvillea rhomboidea, Centratherum punctatum, Sonchus oleraceus, Vernonia scorpioides, Wedelia villosa; Convolvulaceae: Ipomoea sp. 2; Rubiaceae: Borreria verticilata; Scrophulariaceae: Stemodia pratensis; Solanaceae: Solanum paniculatum; Verbenaceae: Priva bahiensis.

Augochlorella sp. - Acanthaceae: Asystasia gangetica.

Augochloropsis cupreola - Acanthaceae: Asystasia gangetica.

Augochloropsis sp. 1 - Boraginaceae: Waltheria indica; Caesalpinaceae: Caesalpinia echinata; Fabaceae: Bowdichia virgilioides; Malpighiaceae: Byrsonima sericea; Rubiaceae: Borreria alata, Diodia sp.; Solanaceae: Solanum paniculatum. 
Anexo I. Continuação.

\section{ESPÉCIE/FAMÍLIA}

Augochloropsis sp. 2 - Rubiaceae: Borreria verticilata.

Augochloropsis sp. 3 - Acanthaceae: Asystasia gangetica.

Dialictus (Chloralictus) sp. 1 - Rubiaceae: Borreria verticilata.

Dialictus (Chloralictus) sp. 2 - Acanthaceae: Asystasia gangetica; Rubiaceae: Borreria verticilata.

Dialictus (Chloralictus) sp. 3. - Rubiaceae: Borreria verticilata.

Dialictus opacus - Acanthaceae: Hygrophila costata, Thunbergia grandiflora; Asteraceae: Centratherum punctatum, Sonchus oleraceus,

Rubiaceae: Borreria verticilata.

Pereirapis semiaurata - Rubiaceae: Borreria alata, Borreria verticilata.

Pseudaugochlora graminea - Acanthaceae: Asystasia gangetica; Caesalpinaceae: Caesalpinia echinata, Senna spectabilis.

Pseudaugochlora pandora - Asteraceae: Baccharis oxyodonta.

Pseudaugochlora flammula - Acanthaceae: Asystasia gangetica.

\section{MEGACHILIDAE}

Coelioxys sp. - Asteraceae: Tilesia baccata.

Dicranthidium arenarium - Fabaceae: Macroptilium latrynoides; Nyctaginaceae: Bougainvillea spectabilis; Schrophulariaceae: Stemodia pratensis.

Megachile (Acentron) lentifera - Schrophulariaceae: Stemodia pratensis.

Megachile (Acentron) sp.1 - Asteraceae: Wedelia villosa; Fabaceae: Machaerium hirtum; Scrophulariaceae: Stemodia pratensis.

Megachile (Acentron) sp.2 - Asteraceae: Tilesia baccata.

Megachile (Austromegachile) sussurans - Asteraceae: Tilesia baccata.

Megachile (Cressoniella) sp. - Scrophulariaceae: Stemodia pratensis.

Megachile (Dactylomegachile) sp. - Scrophulariaceae: Stemodia pratensis.

Megachile (Leptorachis) paulistana - Asteraceae: Tilesia baccata, Vernonia scorpioides.

Megachile (Moureapis) apicipennis - Asteraceae: Vernonia scorpioides.

Megachile (Pseudocentron) sp.1 - Asteraceae: Wedelia villosa; Boraginaceae: Lantana caatingensis.

Megachile (Pseudocentron) sp.2 - Asteraceae: Vernonia scorpioides; Scrophulariaceae: Stemodia pratensis.

Megachile (Pseudocentron) sp.3 - Scrophulariaceae: Stemodia pratensis. 